Research Article

\title{
Boundedness and Global Stability for a Predator-Prey System with the Beddington-DeAngelis Functional Response
}

\author{
Wahiba Khellaf ${ }^{1}$ and Nasreddine Hamri ${ }^{2}$ \\ ${ }^{1}$ Laboratory of LMPA, University of Jijel, BP 98, Ouled Aissa, 18000 Jijel, Algeria \\ ${ }^{2}$ Laboratory Math, University of Mentouri, 25000 Constantine, Algeria \\ Correspondence should be addressed to Wahiba Khellaf, wahiba_khellaf@yahoo.fr \\ Received 17 September 2009; Accepted 19 April 2010 \\ Academic Editor: Bhabani Shankar Dandapat
}

Copyright (C) 2010 W. Khellaf and N. Hamri. This is an open access article distributed under the Creative Commons Attribution License, which permits unrestricted use, distribution, and reproduction in any medium, provided the original work is properly cited.

\begin{abstract}
We study the qualitative behavior of a class of predator-prey models with Beddington-DeAngelistype functional response, primarily from the viewpoint of permanence (uniform persistence). The Beddington-DeAngelis functional response is similar to the Holling type-II functional response but contains a term describing mutual interference by predators. We establish criteria under which we have boundedness of solutions, existence of an attracting set, and global stability of the coexisting interior equilibrium via Lyapunov function.
\end{abstract}

\section{Introduction and Mathematical Model}

Standard Lotka-Volterra systems are also known as the predator-prey systems, on which a large body of existing predator-prey theory is built by assuming that the per capita rate of predation depends on the prey numbers only [1]. Recently, the traditional prey-dependent predator-prey models have been challenged by several biologists based on the fact that functional and numerical response over typical ecological time scale sought to depend on the densities of both prey and predator, especially when predators have to search, share, or compete for food. A more suitable general predator-prey model should be based on the ratio-dependent theory [2-4]. This roughly states that the per capita predator growth rate should be a function of the ratio of prey to predator abundance. Moreover, as the number of predators often changes slowly (relative to prey number), there is often a competition among the predators, and the per capita rate of predation should therefore depend on the numbers of both prey and predator, most probably and simply on their ratios. These hypotheses are strongly supported by numerous field and laboratory experiments and observations [5-9]. 
The general model describing the dynamics of prey-predator populations in continuous time can be written as

$$
\begin{gathered}
\frac{d X}{d \tau}=f(X) X-g(X, Y) Y \\
\frac{d Y}{d \tau}=h(X, Y) Y
\end{gathered}
$$

where $X$ and $Y$ are the densities (or biomasses) of prey and of predators at time $T$, respectively. $f(X)$ is the per capita net prey production in the absence of predation, whereas $g(X, Y)$ is the functional response of predators (the number of preys eaten per predator per unit time). Natural mortality of prey is considered to be negligible compared to mortality due to predation. The function $h(X, Y)$ represents the numerical response of predators (measures the growth rate of predators). The function $f$ will be taken either as the Malthusian growth $f(X)=r X$ or as the logistic model $f(X)=r X(1-X / K)$. The key role in prey-predator models is played by the functional response $g$ (Solomon 1949). Traditionally, it is assumed that the functional response is a function of prey density only prey-dependent feeding, $g=g(X)$, without any dependence on predator density $[9,10]$. The hypothesis is based on an analogy with the law of mass action in chemistry assuming that prey and predator individuals encounter each other randomly in space and time [11]. Therefore, the preydependent model can be applied to systems which are spatially homogeneous and in which the time scale of prey removal by predators is of the same order of magnitude as that of population reproduction [2].

Many questions in predator-prey theory, including the question of interference between predators, revolve around the expression that is used for the functional response $g=$ $g(X, Y)$. Arditi and Ginzburg [2] have argued that, in many cases, this predator dependence could be simplified as a ratio-dependent model $g=g(X / Y)$ instead of modeling explicitly all conceivable interference mechanisms (and thus adding parameters to the model).

The Beddington-DeAngelis-type functional response performed even better. Although the predator-dependent models that they considered fit those data reasonably well, no single functional response best describes all the data sets. The Beddington-DeAngelis response can be generated by a number of natural mechanisms [5, 12], and because it admits rich but biologically reasonable dynamics [6], it is worthy for us to further study the BeddingtonDeAngelis model.

Therefore, it is interesting and important to study the following autonomous predatorprey model with the Beddington-DeAngelis functional response:

$$
\begin{gathered}
\frac{d X}{d \tau}=\left(a_{1}-b_{1} X-\frac{m_{1} Y}{\alpha_{1} X+\beta_{1} Y+\gamma_{1}}\right) X, \\
\frac{d Y}{d \tau}=\left(a_{2}-\frac{m_{2} Y}{X+k_{1}}\right) Y,
\end{gathered}
$$

with the initial values $X(0)>0$ and $Y(0)>0$. The constants $a_{1}, a_{2}, b_{1}, m_{1}, m_{2}, \alpha_{1}, \beta_{1}, \gamma_{1}$, and $k_{1}$ are the parameters of model and are assumed to be nonnegative with $\beta_{1}$ nontrivial (if $\beta_{1}=0$, then the model (1.2) is the same as that in [13]).

These parameters are defined as follows: $a_{1}$ (resp., $a_{2}$ ) describes the growth rate of prey (resp., of predator), $b_{1}$ measures the strength of competition among individuals of 
prey's species, $m_{1}$ is the maximum value which per capita reduction rate of prey can attain, $\gamma_{1}$ (resp., $k_{1}$ ) measures the extent to which environment provides protection to prey (resp., to predator), and $m_{2}$ has a similar meaning to $m_{1}$. The functional response in (1.2) was introduced by Beddington [5] and DeAnglis et al. (1975) in [7]. It is similar to the well-known Holling type-II functional response but has an extra term $\beta_{1} Y$ in the first right term equation modeling mutual interference among predators. Hence this kind of type functional response given in (1.2) is affected by both predator and prey, that is, the so-called predator dependence by Arditi and Ginzburg [2]. Dynamics for the Holling type-II model have been much studied (see, e.g., [13-15]). Then how the mutual interference term affects the dynamic of the whole system is an interesting problem.

Introducing the following scaling (see [16]), $t=a_{1} \tau, x(t)=\left(b_{1} / a_{1}\right) X(\tau)$, and $y(t)=$ $\left(m_{2} b_{1} / a_{1} a_{2}\right) Y(\tau)$, then Beddington-DeAngelis predator-prey model (1.2) should take the following nondimensional form:

$$
\begin{gathered}
\frac{d x}{d t}=x(1-x)-\frac{a x y}{\alpha x+\beta y+\gamma}, \\
\frac{d y}{d t}=b\left(1-\frac{y}{x+k}\right) y,
\end{gathered}
$$

where $a=\left(a_{2} / a_{1}\right)\left(m_{1} / m_{2}\right), b=a_{2} / a_{1}, \alpha=\alpha_{1}, \beta=\beta_{1}\left(a_{2} / m_{2}\right), \gamma=\gamma_{1}\left(b_{1} / a_{1}\right)$, and $k=$ $k_{1}\left(b_{1} / a_{1}\right)$.

\section{Boundedness of the Model and Existence of a Positively Invariant Attracting Set}

We denote by $\mathbb{R}_{+}^{2}$ the nonnegative quadrant, and by $\operatorname{Int}\left(\mathbb{R}_{+}^{2}\right)$ the positive quadrant.

Lemma 2.1. Positive quadrant $\operatorname{Int}\left(\mathbb{R}_{+}^{2}\right)$ is invariant for system (1.3).

Proof. From system (1.3), we observe that the boundaries of the nonnegative quadrant $\mathbb{R}_{+}^{2}$, which are the positives $x$-axis and $y$-axis are invariant; this is immediately obvious from the system (1.3). Therefore, densities $x(t)$ and $y(t)$ are positive for all $t \geq 0$ if $x(0)>0$ and $y(0)>0$. Theorem of existence and uniqueness ensures that the positive solutions of the autonomous system (1.3) and the axis cannot intersect.

Next, we will show that, under some assumptions, the solutions of system (1.3) which start in $\mathbb{R}_{+}^{2}$ are ultimately bounded. First, let us give the following comparison lemma.

Lemma 2.2. Let $\phi$ be an absolutely-continuous function satisfying the differential inequality

$$
\frac{d}{d t} \phi(t)+\alpha_{1} \phi(t) \leq \alpha_{2}, \quad t \geq 0
$$

where $\left(\alpha_{1}, \alpha_{2}\right) \in \mathbb{R}^{2}$; then

$$
\forall t \geq \bar{T}: \phi(t) \leq \frac{\alpha_{1}}{\alpha_{2}}-\left(\frac{\alpha_{1}}{\alpha_{2}}-\phi(\bar{T})\right) e^{-\alpha_{1}(t-\bar{T})}
$$


We define the function $\psi(t)$ to be the solution of differential equation

$$
\frac{d}{d t} \phi(t)+\alpha_{1} \phi(t)=\alpha_{2}, \quad t \geq 0
$$

and we have the Gronwall's lemma.

Lemma 2.3. Let $x(t)$ satisfy for $t \geq t_{0}$ the linear, scalar equation

$$
\begin{gathered}
\frac{d x}{d t}=a(t) x+b(t), \\
x\left(t_{0}\right)=x_{0},
\end{gathered}
$$

with $a(t)$ and $b(t)$ being continuous functions. If $y(t)$ satisfies for $t \geq t_{0}$ the inequalities

$$
\begin{gathered}
\frac{d y}{d t} \leq a(t) x+b(t), \\
y\left(t_{0}\right) \leq x_{0},
\end{gathered}
$$

then

$$
y(t) \leq x(t), \quad t \geq t_{0}
$$

Definition 2.4. A solution $\phi\left(t, t_{0}, x_{0}, y_{0}\right)$ of system (1.3) is said to be ultimately bounded with respect to $\mathbb{R}_{+}^{2}$ if there exists a compact region $A \in \mathbb{R}_{+}^{2}$ and a finite time $T\left(T=T\left(t_{0}, x_{0}, y_{0}\right)\right)$ such that, for any $\left(t_{0}, x_{0}, y_{0}\right) \in \mathbb{R} \times \mathbb{R}_{+}^{2}$,

$$
\phi\left(t, t_{0}, x_{0}, y_{0}\right) \in A, \quad \forall t \geq T
$$

Theorem 2.5. Let $A$ be the set defined by

$$
A=\left\{(x, y) \in \mathbb{R}_{+}^{2}: 0 \leq x \leq 1,0 \leq x+y \leq L_{1}\right\}
$$

where

$$
L_{1}=\frac{1}{4 b}\left\{5 b+(1+b)^{2}(1+k)\right\}
$$

Then

(1) $A$ is positively invariant; and

(2) all solutions of (1.3) initiating in $\mathbb{R}_{+}^{2}$ are ultimately bounded with respect to $\mathbb{R}_{+}^{2}$ and eventually enter the attracting set $A$. 
Proof. Let $(x(0), y(0)) \in A$, we will show that $(x(t), y(t)) \in A$ for all $t \geq 0$. Obviously, from Lemma 2.1, as $(x(0), y(0)) \in A,(x(t), y(t))$ is $\operatorname{in} \operatorname{Int}\left(\mathbb{R}_{+}^{2}\right)$. Then, we have to show that for all $t \geq 0,0 \leq x(t) \leq 1$, and $0 \leq x(t)+y(t) \leq L_{1}$.

(1) (a) First, we prove that for all $t \geq 0,0 \leq x(t) \leq 1$.

We have $x>0$ and $y>0$ in $\operatorname{Int}\left(\mathbb{R}_{+}^{2}\right)$; then every solution $\phi(t)=(x(t), y(t))$ of system (1.3), which starts in $\operatorname{Int}\left(\mathbb{R}_{+}^{2}\right)$, satisfies the differential inequality $d x / d t \leq(1-x(t)) x(t)$. This is obvious by considering the first equation of (1.3). Thus, $x(t)$ may be compared with solutions of $d u / d t=(1-u(t)) u(t)$ and $u(0)=x(0)>0$ which is a Bernoulli's equation; then the solution is $u(t)=1 /\left(1+c e^{-t}\right)$, with $c=1 / x(0)-1$, where $0 \leq x(0) \leq 1$, which implies that $c=1 / x(0)-1 \geq 1$.

It follows that every nonnegative solution $\phi(t)$ satisfies

$$
x(t) \leq 1 \quad \forall t \geq 0 .
$$

(b) We prove now that, for all $t \geq 0,0 \leq x+y \leq L_{1}$.

We define the function $\sigma(t)=x(t)+y(t)$; the time derivative of this function is

$$
\frac{d \sigma}{d t}=\frac{d x}{d t}+\frac{d y}{d t}=\left(1-x-\frac{a y}{\alpha x+\beta y+\gamma}\right) x+b\left(1-\frac{y}{x+k}\right) y
$$

Since all parameters are positive and solutions initiating in $\mathbb{R}_{+}^{2}$ remain in the nonnegative quadrant, then

$$
\frac{d \sigma}{d t} \leq(1-x) x+b\left(1-\frac{y}{x+k}\right) y
$$

holds for all $x$ and $y$ being nonnegative.

Thus, as

$$
\max _{\mathbb{R}_{+}}(1-x) x=\frac{1}{4}
$$

we have

$$
\frac{d \sigma}{d t} \leq \frac{1}{4}+b\left(1-\frac{y}{x+k}\right) y
$$

then

$$
\frac{d \sigma}{d t} \leq \frac{1}{4}+b\left(1-\frac{y}{x+k}\right) y+\sigma(t)-\sigma(t)
$$


which implies that

$$
\frac{d \sigma}{d t}+\sigma(t) \leq \frac{1}{4}+x+\left(b+1-\frac{b y}{x+k}\right) y .
$$

Since in $A, x(t) \leq 1$ for all $t \geq 0$, we obtain

$$
\frac{d \sigma}{d t}+\sigma(t) \leq \frac{5}{4}+\left(b+1-\frac{b y}{1+k}\right) y .
$$

Moreover, it can be easily verified that

$$
\max _{\mathbb{R}_{+}}\left[\left(b+1-\frac{b y}{1+k}\right) y\right]=\frac{1}{4 b}(1+b)^{2}(1+k) .
$$

Consequently

$$
\frac{d \sigma}{d t}+\sigma(t) \leq L_{1}
$$

with

$$
L_{1}=\frac{1}{4 b} 5 b+(1+b)^{2}(1+k) .
$$

Using Lemma 2.2, with

$$
\alpha_{1}=1, \quad \alpha_{2}=L_{1},
$$

then we get

$$
\forall t \geq \bar{T} \geq 0: \sigma(t) \leq L_{1}-\left(L_{1}-\sigma(\bar{T})\right) e^{-(t-\bar{T})} .
$$

Then, if $\bar{T}=0$,

$$
\sigma(t) \leq L_{1}-\left(L_{1}-\sigma(0)\right) e^{-t}
$$

Hence, for $(x(0), y(0)) \in A$,

$$
\sigma(t)=x(t)+y(t) \leq L_{1} .
$$

Then

$$
(x(t), y(t)) \in A, \quad \forall t \geq 0 .
$$


(2) We have to prove that, for $(x(0), y(0)) \in \mathbb{R}_{+}^{2},(x(t), y(t)) \rightarrow A$ when $t \rightarrow+\infty$. We will show that $\varlimsup_{t \rightarrow+\infty} x(t) \leq 1$ and $\varlimsup_{t \rightarrow+\infty}(x(t)+y(t)) \leq L_{1}$.

(a) The first result, $\varlimsup_{t \rightarrow+\infty} x(t) \leq 1$, is obtained directly from (1a) and Lemma 2.2, since solutions of the initial value problem

$$
\frac{d x}{d t}=x(t)(1-x(t)), \quad x(0) \geq 0
$$

verify

$$
\varlimsup_{t \rightarrow+\infty} x(t) \leq 1
$$

(b) For the second result, let $\varepsilon>0$ be given, and $T_{1}>0$ exists, such that

$$
x(t) \leq 1+\frac{\varepsilon}{2} \quad \forall t \geq T_{1} .
$$

From (2.22) with $\bar{T}=T_{1}$, we get, for all $t \geq T_{1}$,

$$
\begin{aligned}
\sigma(t) & =x(t)+y(t) \\
& \leq L_{1}-\left(L_{1}-\sigma\left(T_{1}\right)\right) e^{-\left(t-T_{1}\right)} \\
& \leq L_{1}-\left\{L_{1} e^{T_{1}}-\left(x\left(T_{1}\right)+y\left(T_{1}\right)\right) e^{T_{1}}\right\} e^{-t} \\
& \leq L_{1}-\left\{L_{1}-\left(x\left(T_{1}\right)+y\left(T_{1}\right)\right) e^{T_{1}}\right\} e^{-t} .
\end{aligned}
$$

Then

$$
\sigma(t)=x(t)+y(t) \leq\left(L_{1}+\frac{\varepsilon}{2}\right)-\left\{\left(L_{1}+\frac{\varepsilon}{2}\right)-\left(x\left(T_{1}\right)+y\left(T_{1}\right)\right) e^{T_{1}}\right\} e^{-t}, \quad \forall t \geq T_{1} \geq 0
$$

Let $T_{2} \geq T_{1}$ be such that

$$
\left(L_{1}+\frac{\varepsilon}{2}\right)-\left(x\left(T_{1}\right)+y\left(T_{1}\right)\right) e^{T_{1}} e^{-t} \leq \frac{\varepsilon}{2}, \quad \forall t \geq T_{2} .
$$

Then

$$
x(t)+y(t) \leq L_{1}+\varepsilon \quad \forall t \geq T_{2} .
$$

Hence,

$$
\varlimsup_{t \rightarrow+\infty}(x(t)+y(t)) \leq L_{1} .
$$


This completes the proof; then we conclude that system (1.3) is dissipative in $\mathbb{R}_{+}^{2}$.

\section{Linear Stability}

First of all, it is easy to verify that this system has three trivial equilibria, (belonging to the boundary of $\mathbb{R}_{+}^{2}$, i.e., at which one or more of populations has zero density or is extinct)

$$
P_{1}(0,0), \quad P_{2}(0, k), \quad P_{3}(1,0)
$$

The other equilibria are defined by the system

$$
\begin{gathered}
\frac{a y}{\alpha x+\beta y+\gamma}=1-x, \\
y=x+k .
\end{gathered}
$$

Proposition 3.1. The system (1.3) has a unique interior equilibria $P^{*}\left(x^{*}, y^{*}\right)$ (i.e., $x^{*}>0$ and $y^{*}>0$ ) if the following condition is verified:

$$
k(a-\beta) \leq \gamma
$$

Proof. We introduce the second equation of (3.2) in the first one; then

$$
a(x+k)=(1-x)((\alpha+\beta) x+(\beta k+\gamma))
$$

and we obtain

$$
(\alpha+\beta) x^{2}+(\beta k+\gamma+a-\alpha-\beta) x+a k-\beta k-\gamma=0 .
$$

The discriminant of this equation

$$
\begin{aligned}
\Delta= & (\beta k+\gamma+a-\alpha-\beta)^{2}-4(a k-\beta k-\gamma)(\alpha+\beta) \\
= & ((\beta k+\gamma+a)-(\alpha+\beta))^{2}+4((\beta k+\gamma)-a k)(\alpha+\beta) \\
= & (\beta k+\gamma+a)^{2}+(\alpha+\beta)^{2}-2(\beta k+\gamma+a)(\alpha+\beta)+4(\beta k+\gamma+a)(\alpha+\beta) \\
& -4 a(k+1)(\alpha+\beta) \\
= & ((\beta k+\gamma+a)+(\alpha+\beta))^{2}-4 a(1+k)(\alpha+\beta) .
\end{aligned}
$$


Therefore, if (3.3) holds, then

$$
\begin{aligned}
\Delta & =((\beta k+\gamma)+a+(\alpha+\beta))^{2}-4 a(1+k)(\alpha+\beta) \\
& \geq(a(k+1)+(\alpha+\beta))^{2}-4 a(1+k)(\alpha+\beta) \\
& \geq(a(k+1)-(\alpha+\beta))^{2} \geq 0 .
\end{aligned}
$$

Consequently, $\Delta$ is positive, and the system (1.3) has two other equilibriums $P_{1}^{*}\left(x_{1}, y_{1}\right)$ and $P_{2}^{*}\left(x_{2}, y_{2}\right)$, where

$$
\begin{gathered}
x_{1,2}=\frac{((\beta k+\gamma)-(\alpha+\beta)+a) \pm \sqrt{\Delta}}{2(\alpha+\beta)}, \\
y_{1,2}=\left(x_{1,2}+k\right) .
\end{gathered}
$$

Now, we show, under the condition (3.3), that one of this equilibriums is not in $\mathbb{R}_{+}^{2}$; let

$$
x_{2}=\frac{((\beta k+\gamma+a)-(\alpha+\beta))-\sqrt{\Delta}}{2(\alpha+\beta)} ;
$$

then

$$
2 x_{2}=1-\frac{(\beta k+\gamma+a)+\sqrt{\Delta}}{(\alpha+\beta)},
$$

from (3.7)

$$
\sqrt{\Delta} \geq|a(k+1)-(\alpha+\beta)|
$$

and due to (3.3)

$$
2 x_{2} \leq 1-\frac{(a(k+1)+|a(k+1)-(\alpha+\beta)|)}{(\alpha+\beta)},
$$

which implies that

(1) if $a(k+1)<(\alpha+\beta)$

$$
2 x_{2} \leq 1-\frac{a(k+1)-a(k+1)+(\alpha+\beta)}{(\alpha+\beta)} \leq 0
$$


(2) if $a(k+1)>(\alpha+\beta)$,

$$
2 x_{2} \leq 1-\frac{a(k+1)+a(k+1)-(\alpha+\beta)}{(\alpha+\beta)} \leq 2-2 \frac{a(k+1)}{\alpha+\beta} \leq 0 .
$$

it results that $P_{2}^{*}\left(x_{2}, y_{2}\right)$ is not in $\mathbb{R}_{+}^{2}$, such that

$$
x_{1} x_{2}=a k-\beta k-\gamma \leq 0
$$

then the first point $P_{1}^{*}\left(x_{1}, y_{1}\right)$ is in $\mathbb{R}_{+}^{2}$.

The Jacobian matrix is given by

$$
J\left(X_{i}\right)=\left(\begin{array}{ll}
\frac{\partial f_{1}}{\partial x}\left(x_{i}, y_{i}\right) & \frac{\partial f_{1}}{\partial y}\left(x_{i}, y_{i}\right) \\
\frac{\partial f_{2}}{\partial x}\left(x_{i}, y_{i}\right) & \frac{\partial f_{2}}{\partial y}\left(x_{i}, y_{i}\right)
\end{array}\right)
$$

where

$$
\begin{aligned}
& \frac{\partial f_{1}}{\partial x}\left(x_{i}, y_{i}\right)=1-2 x-\frac{a y(\beta y+\gamma)}{(\alpha x+\beta y+\gamma)^{2}} \\
& \frac{\partial f_{1}}{\partial y}\left(x_{i}, y_{i}\right)=-\frac{a x(\alpha x+\gamma)}{(\alpha x+\beta y+\gamma)^{2}} \\
& \frac{\partial f_{2}}{\partial x}\left(x_{i}, y_{i}\right)=b\left(\frac{y}{x+k}\right)^{2} \\
& \frac{\partial f_{2}}{\partial y}\left(x_{i}, y_{i}\right)=b-\frac{2 b y}{x+k} .
\end{aligned}
$$

(1) At $P_{0}(0,0)$,

$$
J\left(P_{0}\right)=\left(\begin{array}{ll}
1 & 0 \\
0 & b
\end{array}\right) .
$$

The eigenvalues of this matrix are

$$
\lambda_{1}=1, \quad \lambda_{2}=b .
$$

Hence, all parameters are positive; then $P_{0}(0,0)$ is an unstable node. 
(2) At $P_{1}(0, k)$,

$$
J\left(P_{1}\right)=\left(\begin{array}{cc}
1-\frac{a k}{\beta k+\gamma} & 0 \\
b & -b
\end{array}\right) .
$$

The eigenvalues are

$$
\lambda_{1}=1-\frac{a k}{\beta k+\gamma}, \quad \lambda_{2}=-b<0 .
$$

then, we have

(a) if $k(a-\beta)>\gamma, P_{1}(0, k)$ is stable node;

(b) if $k(a-\beta) \leq \gamma, P_{1}(0, k)$ is unstable with the positive $y$-axis as its stable manifold.

(3) At $P_{2}(1,0)$,

$$
J\left(P_{2}\right)=\left(\begin{array}{cc}
-1 & -\frac{a}{\alpha+\gamma} \\
0 & b
\end{array}\right) .
$$

The eigenvalues are

$$
\lambda_{1}=-1<0, \quad \lambda_{2}=b>0 .
$$

Then the equilibrium $P_{2}(1,0)$ is a saddle point with the stable manifold being the $x$-axis.

Around $P^{*}\left(x^{*}, y^{*}\right)$, the Jacobian matrix takes the form

$$
J\left(P^{*}\right)=\left(\begin{array}{cc}
1-2 x^{*}-\frac{a y^{*}\left(\beta y^{*}+\gamma\right)}{\left(\alpha x^{*}+\beta y^{*}+\gamma\right)^{2}} & -\frac{a x^{*}\left(\alpha x^{*}+\gamma\right)}{\left(\alpha x^{*}+\beta y^{*}+\gamma\right)^{2}} \\
b & -b
\end{array}\right) .
$$

The characteristic equation is

$$
\lambda^{2}-\operatorname{tr} J\left(P^{*}\right) \mathcal{\lambda}+\operatorname{det} J\left(P^{*}\right)=0,
$$


where

$$
\begin{gathered}
\operatorname{det} J\left(x^{*}, y^{*}\right)=-b\left(1-2 x^{*}-\frac{a y^{*}\left(\beta y^{*}+\gamma\right)}{\left(\alpha x^{*}+\beta y^{*}+\gamma\right)^{2}}\right)+a b \frac{x^{*}\left(\alpha x^{*}+\gamma\right)}{\left(\alpha x^{*}+\beta y^{*}+\gamma\right)^{2}} \\
=\frac{b}{\left(\alpha x^{*}+\beta y^{*}+\gamma\right)^{2}}\left\{a y^{*}\left(\beta y^{*}+\gamma\right)+\left(2 x^{*}-1\right)\left(\alpha x^{*}+\beta y^{*}+\gamma\right)^{2}\right. \\
\left.\quad+a x^{*}\left(\alpha x^{*}+\gamma\right)\right\} \\
=\frac{b \quad\left\{\left(x^{*}-1\right)\left(\alpha x^{*}+\beta y^{*}+\gamma\right)^{2}+x^{*}\left(\alpha x^{*}+\beta y^{*}+\gamma\right)^{2}\right.}{\left(\alpha x^{*}+\beta y^{*}+\gamma\right)^{2}} \\
\left.\quad+a y^{*}\left(\beta y^{*}+\gamma\right)+a x^{*}\left(\alpha x^{*}+\gamma\right)\right\} .
\end{gathered}
$$

From (3.2), we get

$$
\begin{aligned}
\operatorname{det} J\left(P^{*}\right)= & \frac{b}{\left(\alpha x^{*}+\beta y^{*}+\gamma\right)^{2}}\left\{-a y^{*}\left(\alpha x^{*}+\beta y^{*}+\gamma\right)+x^{*}\left(\alpha x^{*}+\beta y^{*}+\gamma\right)^{2}\right. \\
& \left.+a y^{*}\left(\beta y^{*}+\gamma\right)+a x^{*}\left(\alpha x^{*}+\gamma\right)\right\} \\
= & \frac{b}{\left(\alpha x^{*}+\beta y^{*}+\gamma\right)^{2}}\left\{-a \alpha x^{*} y^{*}+x^{*}\left(\alpha x^{*}+\beta y^{*}+\gamma\right)^{2}+a x^{*}\left(\alpha x^{*}+\gamma\right)\right\} \\
= & \frac{b}{\left(\alpha x^{*}+\beta y^{*}+\gamma\right)^{2}}\left\{x^{*}\left(\alpha x^{*}+\beta y^{*}+\gamma\right)^{2}+a x^{*}\left(\alpha x^{*}-\alpha y^{*}+\gamma\right)\right\} \\
= & \frac{b}{\left(\alpha x^{*}+\beta y^{*}+\gamma\right)^{2}}\left\{x^{*}\left(\alpha x^{*}+\beta y^{*}+\gamma\right)^{2}-a x^{*}(\alpha k-\gamma)\right\}
\end{aligned}
$$

then

$$
\operatorname{det} J\left(P^{*}\right)=\frac{b x^{*}}{\left(\alpha x^{*}+\beta y^{*}+\gamma\right)^{2}}\left\{\left(\alpha x^{*}+\beta y^{*}+\gamma\right)^{2}-a(\alpha k-\gamma)\right\} \text {. }
$$

We observe that det $J\left(P^{*}\right)$ is positive if

$$
\left\{\left(\alpha x^{*}+\beta y^{*}+\gamma\right)^{2}-a(\alpha k-\gamma)\right\}>0 .
$$


To simplify, we developed det $J\left(P^{*}\right)$ respecting one variable, from (3.2); then

$$
\begin{aligned}
\left(\alpha x^{*}+\beta y^{*}+\gamma\right)^{2}-a(\alpha k-\gamma) & =\left(\frac{a\left(x^{*}+k\right)}{1-x^{*}}\right)^{2}-a(\alpha k-\gamma) \\
& =\frac{a^{2}\left(x^{*}+k\right)^{2}}{\left(1-x^{*}\right)^{2}}-a(\alpha k-\gamma)
\end{aligned}
$$

which implies that det $J\left(P^{*}\right)$ has the same sign of

$$
a\left(x^{*}+k\right)^{2}+\left(1-x^{*}\right)^{2}(\gamma-\alpha k) \text {. }
$$

We rewrite

$$
a\left(x^{*}\right)^{2}+2 k a x^{*}+a k^{2}+(\gamma-\alpha k)\left(\left(x^{*}\right)^{2}-2 x^{*}+1\right) .
$$

Let

$$
f(x)=(a+\gamma-\alpha k) x^{2}+2(a k+\alpha k-\gamma) x+\left(\gamma-\alpha k+a k^{2}\right) \text {. }
$$

The discriminant is

$$
\begin{aligned}
\Delta^{\prime}= & (a k+\alpha k-\gamma)^{2}-(a+\gamma-\alpha k)\left(\gamma-\alpha k+a k^{2}\right) \\
= & (a+\alpha)^{2} k^{2}-2 \gamma k(a+\alpha)+\gamma^{2}-(a+\gamma)\left(a k^{2}+\gamma\right)+\left(a k^{2}+a+2 \gamma\right) \alpha k-(\alpha k)^{2} \\
= & \left(a^{2}+2 a \alpha+\alpha^{2}\right) k^{2}-2 \gamma k(a+\alpha)+\gamma^{2}-\left(a^{2} k^{2}+a\left(1+k^{2}\right) \gamma+\gamma^{2}\right)+a \alpha k^{3}+\gamma \\
& +(a+2 \gamma) \alpha k-\alpha^{2} k^{2} \\
= & 2 a \alpha k^{2}-2 a \gamma k-a \gamma k^{2}-a \gamma+a \alpha k^{3}+a \alpha k \\
= & a\left\{\alpha k^{3}+(2 \alpha-\gamma) k^{2}+(\alpha-\gamma) k-\gamma\right\} \\
= & a\left\{\alpha k\left(k^{2}+2 k+1\right)-\gamma\left(k^{2}+2 k+1\right)\right\} \\
= & a(\alpha k-\gamma)(k+1)^{2} .
\end{aligned}
$$

We get three cases.

(1) If $\alpha k<\gamma, \Delta^{\prime}$ is negative, $f(x)$ has the same sign of $(a+\gamma-\alpha k)$, and we have $(a+\gamma-\alpha k)>a>0$.

Then, det $J\left(P^{*}\right)$ is positive. 
(2) If $\alpha k>\gamma, \Delta^{\prime}$ is positive and $f(x)$ has at list two solutions $x_{1}$ and $x_{2}$, then

(a) if $\left.x \in]-\infty, x_{1}\right] \cup\left[x_{2},+\infty[, f(x)\right.$ has the same sign of $(a+\gamma-\alpha k)$;

(b) if $x \in] x_{1}, x_{2}$ [ has sign of $-(a+\gamma-\alpha k)$.

(3) If $\alpha k=\gamma$, then

$$
f(x)=a x^{2}+2 a k x+a k^{2}=a(x+k)^{2}>0 .
$$

Then, det $J\left(P^{*}\right)$ is positive.

Remark 3.2. From the expression (3.28), we find that $\operatorname{det}\left(J\left(P^{*}\right)\right)$ is positive, if $\alpha k \leq r$, hence the eigenvalues associated to $P^{*}$ have the same sign.

To determine the sign of these eigenvalues, we have

$$
\begin{aligned}
\operatorname{tr} J\left(P^{*}\right)= & 1-2 x^{*}-\frac{a y^{*}\left(\beta y^{*}+\gamma\right)}{\left(\alpha x^{*}+\beta y^{*}+\gamma\right)^{2}}-b \\
= & \frac{1}{\left(\alpha x^{*}+\beta y^{*}+\gamma\right)^{2}}\left\{\left(1-2 x^{*}-b\right)\left(\alpha x^{*}+\beta y^{*}+\gamma\right)^{2}-a y^{*}\left(\beta y^{*}+\gamma\right)\right\} \\
= & \frac{1}{\left(\alpha x^{*}+\beta y^{*}+\gamma\right)^{2}}\left\{\left(1-x^{*}\right)\left(\alpha x^{*}+\beta y^{*}+\gamma\right)^{2}-\left(x^{*}+b\right)\left(\alpha x^{*}+\beta y^{*}+\gamma\right)^{2}\right. \\
& \left.\quad-a y^{*}\left(\beta y^{*}+\gamma\right)\right\} .
\end{aligned}
$$

From (3.2), we get

$$
\begin{aligned}
\operatorname{tr} J\left(P^{*}\right)= & \frac{1}{\left(\alpha x^{*}+\beta y^{*}+\gamma\right)^{2}}\left\{a y^{*}\left(\alpha x^{*}+\beta y^{*}+\gamma\right)-\left(x^{*}+b\right)\left(\alpha x^{*}+\beta y^{*}+\gamma\right)^{2}\right. \\
& \left.\quad-a y^{*}\left(\beta y^{*}+\gamma\right)\right\} \\
= & \frac{1}{\left(\alpha x^{*}+\beta y^{*}+\gamma\right)^{2}}\left\{a \alpha x^{*} y^{*}-\left(x^{*}+b\right)\left(\alpha x^{*}+\beta y^{*}+\gamma\right)^{2}\right\} \\
= & \alpha x^{*} \frac{a y^{*}}{\left(\alpha x^{*}+\beta y^{*}+\gamma\right)^{2}}-\left(x^{*}+b\right) \\
= & \alpha x^{*} \frac{a y^{*}\left(1-x^{*}\right)^{2}}{a^{2}\left(y^{*}\right)^{2}}-\left(x^{*}+b\right)
\end{aligned}
$$




$$
\begin{aligned}
& =\alpha x^{*} \frac{\left(1-x^{*}\right)^{2}}{a y^{*}}-\left(x^{*}+b\right) \\
& =\frac{1}{a\left(x^{*}+k\right)}\left\{\alpha x^{*}\left(1-x^{*}\right)^{2}-a\left(x^{*}+b\right)\left(x^{*}+k\right)\right\} \\
& =\frac{1}{a\left(x^{*}+k\right)}\left\{\alpha\left(x^{*}\right)^{3}-(a+2 \alpha)\left(x^{*}\right)^{2}-(a(b+k)-\alpha) x^{*}-a b k\right\} .
\end{aligned}
$$

Let

$$
P_{3}(x)=\alpha x^{3}-(a+2 \alpha) x^{2}-(a(b+k)-\alpha) x-a b k
$$

We obtain the following lemma.

Lemma 3.3. If $\alpha k<\gamma$ is verified, the interior equilibrium $P^{*}\left(x^{*}, y^{*}\right)$ is locally asymptotically stable if $P_{3}\left(x^{*}\right)>0$ and it is unstable if $P_{3}\left(x^{*}\right)<0$.

We used the Cardan's method to solve the cubic equation $P_{3}(x)=0$. Then we consider the equation

$$
a_{3} x^{3}+a_{2} x^{2}+a_{1} x+a_{0}=0
$$

with $a_{3}=\alpha, a_{2}=-(a+2 \alpha), a_{1}=-(a(b+k)-\alpha)$, and $a_{0}=a b k$. Making the substitution $y=a_{3} x+a_{2} / 3$ reduces the equation to the standard form

$$
y^{3}-p y-q=0,
$$

where $p$ and $q$ depend on $a_{3}, a_{2}, a_{1}$, and $a_{0}$

$$
\begin{gathered}
p=-a_{1} a_{3}+\frac{a_{2}^{2}}{3} \\
q=-a_{0} a_{3}^{2}-2\left(\frac{a_{2}}{3}\right)^{3}+\frac{a_{3} a_{2} a_{1}}{3} .
\end{gathered}
$$

Let

$$
y=u+v .
$$

Then

$$
y^{3}-p y-q=(u+v)^{3}-3 u v(u+v)-\left(u^{3}+v^{3}\right)=0 .
$$


We get after the identification of the coefficients

$$
\begin{gathered}
3 u v=p \\
u^{3}+v^{3}=q .
\end{gathered}
$$

Then

$$
\begin{gathered}
u^{3} v^{3}=\left(\frac{p}{3}\right)^{3} \\
u^{3}+v^{3}=q
\end{gathered}
$$

and we obtain that $u^{3}$ and $v^{3}$ are solutions of the quadratic equation

$$
z^{2}-q z+\left(\frac{p}{3}\right)^{3}=0
$$

Then we constitute three cases.

(1) if $27 q^{2}-4 p^{3}>0$, then

$$
\begin{aligned}
& u^{3}=\frac{q}{2}+\sqrt{\left(\frac{q}{2}\right)^{2}-\left(\frac{p}{3}\right)^{3}} \\
& v^{3}=\frac{q}{2}-\sqrt{\left(\frac{q}{2}\right)^{2}-\left(\frac{p}{3}\right)^{3}} .
\end{aligned}
$$

We have

$$
y=\sqrt[3]{\frac{q}{2}-\frac{1}{2} \sqrt{\frac{27 q^{2}-4 p^{3}}{27}}}+\sqrt[3]{\frac{q}{2}+\frac{1}{2} \sqrt{\frac{27 q^{2}-4 p^{3}}{27}}}
$$

then (3.39) has at list one real root

$$
r_{0}=\frac{1}{a_{3}}\left(\sqrt[3]{\frac{q}{2}-\frac{1}{2} \sqrt{\frac{27 q^{2}-4 p^{3}}{27}}}+\sqrt[3]{\left.\frac{q}{2}+\frac{1}{2} \sqrt{\frac{27 q^{2}-4 p^{3}}{27}}-\frac{a_{2}}{3}\right)}\right.
$$

So, we have $P_{3}(x)<0$ if $0<x<r_{0}$, and $P_{3}(x)>0$ if $r_{0}<x$. 
(2) if $27 q^{2}-4 p^{3}<0$, then there are three real roots $r_{1}, r_{2}$, and $r_{3}$, and therefore, $r_{1} r_{2} r_{3}=$ $a b k>0$ then one of them is positive, then

(a) if $r_{2}<r_{3}<0<r_{1}$, then $P_{3}(x)>0$ if $0<r_{1}<x$, and $P_{3}(x)<0$ if $x<r_{1}$;

(b) if $0<r_{1}<r_{2}<r_{3}$, then $P_{3}(x)>0$ if $0<r_{1}<x$, and $P_{3}(x)<0$ if $x<r_{1}$.

(3) if $27 q^{2}=4 p^{3}$, then there are one real root positive $r_{0}=2 \sqrt[3]{q / 2}$ and a double root $r_{1,2}=-\sqrt[3]{q / 2}$; we also have $P_{3}(x)<0$ if $0<x<r_{0}$, and $P_{3}(x)>0$ if $r_{0}<x$.

\section{Uniform Permanence}

In this section we shall prove the permanence $[8,17-19]$, that is, the uniform persistence and dissipativity, of system (1.3).

The principal notion of persistence theory is uniform persistence or permanence. Before the study of the permanence of system (1.3), we introduce some necessary definitions. Consider an ODE model for $\mathrm{n}$ interacting biological species

$$
\frac{d x_{i}}{d t}=f_{i}\left(x_{1}, x_{2}, \ldots, x_{n}\right), \quad i=1,2, \ldots, n
$$

where $x_{i}(t)$ denotes the density of the ith species. Let $\left(x_{1}(t), x_{2}(t), \ldots, x_{n}(t)\right)$ denote the solution of (4.1) with componentwise positive initial values. The system (4.1) is said to be weakly persistent if

$$
\limsup _{t \rightarrow+\infty} x_{i}(t)>0, \quad i=1,2, \ldots, n,
$$

persistent if

$$
\liminf _{t \rightarrow+\infty} x_{i}(t)>0, \quad i=1,2, \ldots, n,
$$

and uniformly persistent if there is an $\varepsilon_{0}>0$ such that

$$
\liminf _{t \rightarrow+\infty} x_{i}(t) \geq \varepsilon_{0}, \quad i=1,2, \ldots, n
$$

The system (4.1) is said to be permanent if for each $i=1,2, \ldots, n$ there are constants $\varepsilon_{0}$ and $M_{i}$ such that

$$
0<\varepsilon_{0} \leq \liminf _{t \rightarrow+\infty} x_{i}(t) \leq \limsup _{t \rightarrow+\infty} x_{i}(t) \leq M_{i}
$$

Clearly, a permanent system is uniformly persistent which in turn is persistent, and persistence implies weak persistence; a dissipative uniformly persistent system is permanent. For further discussion about various definitions of persistence and permanence and their connections, see [18]. 
Suppose that $Y$ is a complete metric space with $Y=Y_{0} \cup \partial Y_{0}$ for an open set $Y_{0}$. We will choose $Y_{0}$ to be the positive cone in $\mathbb{R}^{2}$. For the following definitions and theorems, one can see [6], and for the proof of the theorem, see [8].

Definition 4.1. A flow or semiflow on $Y$ under which $Y_{0}$ and $\partial Y_{0}$ are forward invariant is said to be permanent if it is dissipative and if there is a number $\varepsilon>0$ such that any trajectory starting in $Y_{0}$ will be at least a distance $\varepsilon$ from $\partial Y_{0}$ for all sufficiently large $t$.

Let $\omega\left(\partial Y_{0}\right) \in \partial Y_{0}$ denote the union of the sets $\omega(u)$ over $u \in \partial Y_{0}$.

Definition 4.2. The $\omega$-limit set $\omega\left(\partial Y_{0}\right)$ is said to be isolated if it has a covering $\Omega=\bigcup_{k=1}^{N} \Omega_{k}$ of pairwise disjoint sets $\Omega_{k}$ which are isolated and invariant with respect to the flow or the semiflow both on $\partial Y_{0}$ and on $Y=Y_{0} \cup \partial Y_{0}$, ( $M$ is called an isolated covering). The set $\omega\left(\partial Y_{0}\right)$ is said to be acyclic if there exists an isolated covering $\bigcup_{k=1}^{N} \Omega_{k}$ such that no subset of $\Omega_{k}$ is a cycle.

Theorem 4.3 (Hale and Waltman 1989). Suppose that a semiflow on $Y$ leaves both $Y_{0}$ and $\partial Y_{0}$ forward invariant, maps bounded sets in $Y$ to precompact set for $t>0$, and it is dissipative. If in addition

(1) $\omega\left(\partial Y_{0}\right)$ is isolated and acyclic;

(2) $W^{s}\left(\Omega_{k}\right) \cap Y_{0}=\emptyset$ for all $K$, where $\bigcup_{k=1}^{N} \Omega_{k}$ is the isolated covering used in the definition of acyclicity of $\partial Y_{0}$, and $W^{s}$ denote the stable manifold.

Then the semiflow is permanent.

And, we have this theorem.

Theorem 4.4. Let us assume the following condition:

$$
k(a-\beta) \leq \gamma
$$

Then, system (1.3) is permanent.

Proof. We take $Y$ the strictly positive quadrant of $R^{2}$; then $\omega\left(\partial Y_{0}\right)$ consists of the equilibria $P_{0}(0,0), P_{1}(0, k)$, and $P_{2}(1,0) . P_{0}(0,0)$ is an unstable node, $P_{2}(1,0)$ is saddle point, and its stable manifold is $x$-axis. If $a k \leq \beta k+\gamma, P_{1}(0, k)$ is a saddle point stable along the $y$-axis and unstable along the $x$-axis.

Then, all trajectories on the axis $(o x)$ other than $P_{0}(0,0)$ approach the point $P_{2}(1,0)$ and all trajectories on the axis $(o y)$ other than $P_{0}(0,0)$ approach the point $P_{1}(0, k)$. It follows from these structural features that the flow in $\partial Y_{0}$ is acyclic. So $\omega\left(\partial Y_{0}\right)$ is isolated and acyclic. The stable manifold of $P_{2}(1,0)$ is the $x$-axis and the stable manifold of $P_{1}(0, k)$ is the $y$-axis, and we know, from Theorem 2.5, that these stable manifolds cannot intersect the interior of $Y_{0}$.

In this case, Theorem 4.3 implies permanence of the flow defined by (1.3). 


\section{Global Stability}

In this section, we shall prove the global stability of system (1.3) by constructing a suitable Lyapunov function. First, we have to show that there exists one interior equilibrium $P^{*}\left(x^{*}, y^{*}\right)$. The linear analysis shows that if $\alpha k \leq \gamma$ and $x^{*}<x(0)$, then $P^{*}\left(x^{*}, y^{*}\right)$ is locally stable. We prove now that, under some assumptions, this steady state is globally asymptotically stable.

Theorem 5.1. The interior equilibrium $P^{*}\left(x^{*}, y^{*}\right)$ is globally asymptotically stable if

$$
\begin{gathered}
\beta<\alpha, \\
2 a L_{1}<\gamma, \\
(\beta k+\gamma)\left(\alpha L_{1}+\gamma\right)(1+k)<4 \beta k^{2} \gamma^{2}, \\
\alpha(1+2 k)-\beta(1+k)<\gamma, \\
a-4 \beta L_{1}<4 \gamma .
\end{gathered}
$$

Proof. The proof is based on construction of a positive definite Lyapunov function. Let

$$
V(x, y)=V_{1}(x, y)+V_{2}(x, y)
$$

where

$$
\begin{aligned}
& V_{1}(x, y)=\left(\alpha x^{*}+\beta y^{*}+\gamma\right)\left(x-x^{*}-x^{*} \ln \left(\frac{x}{x^{*}}\right)\right), \\
& V_{2}(x, y)=\frac{a}{b}\left(x^{*}+k\right)\left(y-y^{*}-y^{*} \ln \left(\frac{y}{y^{*}}\right)\right) .
\end{aligned}
$$

This function is defined and continuous on $\operatorname{Int}\left(\mathbb{R}_{+}^{2}\right)$.

We can easily verify that the function $V(x, y)$ is zero at the equilibrium $\left(x^{*}, y^{*}\right)$ and is positive for all other positive values of $x$ and $y$, and thus, $P^{*}\left(x^{*}, y^{*}\right)$ is the global minimum of $V$.

Since the solutions of (1.3) are bounded and ultimately enter the set $A$, we restrict the study for this set. The time derivative of $V_{1}$ and $V_{2}$ along the solutions of system (1.3) is

$$
\frac{d V_{i}}{d t}=\frac{d V_{i}}{d x} \dot{x}+\frac{d V_{i}}{d y} \dot{y}, \quad i=1,2
$$

Then

$$
\begin{aligned}
& \frac{d V_{1}}{d t}(x, y)=\left(\alpha x^{*}+\beta y^{*}+\gamma\right)\left(1-\frac{x^{*}}{x}\right) \dot{x}, \\
& \frac{d V_{2}}{d t}(x, y)=\frac{a}{b}\left(x^{*}+k\right)\left(1-\frac{y^{*}}{y}\right) \dot{y}
\end{aligned}
$$


and using (3.2), we get after simplifing

$$
\begin{aligned}
\frac{d V_{1}}{d t}(x, y)= & -\left(\alpha x^{*}+\beta y^{*}+\gamma\right)\left(x-x^{*}\right)^{2}-\left(x-x^{*}\right)\left(y-y^{*}\right)\left(\frac{a \alpha x+a \gamma}{\alpha x+\beta y+\gamma}\right) \\
& +\left(\frac{a \alpha y}{\alpha x+\beta y+\gamma}\right)\left(x-x^{*}\right)^{2}, \\
\frac{d V_{2}}{d t}(x, y)= & -a\left(y-y^{*}\right)^{2}+\frac{a y}{x+k}\left(x-x^{*}\right)\left(y-y^{*}\right) .
\end{aligned}
$$

Therefore, computing $d V / d t$ via $d V_{1} / d t$ and $d V_{2} / d t$ yields

$$
\begin{aligned}
\frac{d V}{d t}= & \left(-\left(\alpha x^{*}+\beta y^{*}+\gamma\right)+\left(\frac{a \alpha y}{\alpha x+\beta y+\gamma}\right)\right)\left(x-x^{*}\right)^{2} \\
& +\left(x-x^{*}\right)\left(y-y^{*}\right)\left(-\left(\frac{a \alpha x+a \gamma}{\alpha x+\beta y+\gamma}\right)+\frac{a y}{x+k}\right)-a\left(y-y^{*}\right)^{2} .
\end{aligned}
$$

The above equation can be written as

$$
\frac{d V}{d t}(x, y)=-\left(x-x^{*}, y-y^{*}\right)\left(\begin{array}{cc}
-g(x, y) & -h(x, y) \\
-h(x, y) & a
\end{array}\right)\left(\begin{array}{c}
x-x^{*} \\
y-y^{*}
\end{array}\right)
$$

where

$$
\begin{gathered}
g(x, y)=-\left(\alpha x^{*}+\beta y^{*}+\gamma\right)+\frac{a \alpha y}{\alpha x+\beta y+\gamma} \\
h(x, y)=\frac{a}{2}\left(\frac{-\alpha x+\gamma}{\alpha x+\beta y+\gamma}+\frac{y}{x+k}\right) .
\end{gathered}
$$

From (5.11), it is obvious that $d V / d t<0$ if the matrix above is positive definite. This matrix is positive definite if only if all upper-left submatrices are positive (Sylvester's criteria), that is, since $a>0$, if only if
(1) $g(x, y)<0$;
(2) $\phi(x, y)=a g(x, y)+h^{2}(x, y)<0$. 
Proof. It is of (1)

$$
g(x, y)=-\left(\alpha x^{*}+\beta y^{*}+\gamma\right)+\frac{a \alpha y}{\alpha x+\beta y+\gamma}<0
$$

So, as $A$ is an attracting positively invariant set, where, all solutions satisfy $0 \leq x \leq 1$ and $0 \leq x+y \leq L_{1}$, then

$$
\begin{gathered}
g(x, y) \leq-\alpha x^{*}+\frac{a \alpha y}{\alpha x+\beta y+\gamma}, \\
g(x, y) \leq \alpha\left(-1+\frac{a y^{*}}{\alpha x^{*}+\beta y^{*}+\gamma}+\frac{a y}{\alpha x+\beta y+\gamma}\right), \\
g(x, y) \leq \alpha\left(-1+\frac{2 a L_{1}}{\gamma}\right) .
\end{gathered}
$$

Therefore, if (5.2) holds, then

$$
g(x, y)<0, \quad \forall(x, y) \in A .
$$

Proof. It is of (2)

$$
\phi(x, y)=-a\left(\alpha x^{*}+\beta y^{*}+\gamma\right)+\frac{a^{2} \alpha y}{\alpha x+\beta y+\gamma}+\frac{a^{2}}{4}\left(-\frac{\alpha x+\gamma}{\alpha x+\beta y+\gamma}+\frac{y}{x+k}\right)^{2}<0 .
$$

Since (for $x$ fixed)

$$
\frac{\partial \phi}{\partial y}=\frac{a^{2} \alpha(\alpha x+\gamma)}{(\alpha x+\beta y+\gamma)^{2}}+\frac{a^{2}}{2}\left(-\frac{\alpha x+\gamma}{\alpha x+\beta y+\gamma}+\frac{y}{x+k}\right)\left(\frac{\beta(\alpha x+\gamma)}{(\alpha x+\beta y+\gamma)^{2}}+\frac{1}{x+k}\right)
$$

then

$$
\begin{aligned}
\frac{\partial^{2} \phi}{\partial y^{2}}= & -2 a^{2} \frac{\alpha \beta(\alpha x+\gamma)}{(\alpha x+\beta y+\gamma)^{3}}+\frac{a^{2}}{2}\left(\frac{\beta(\alpha x+\gamma)}{(\alpha x+\beta y+\gamma)^{2}}+\frac{1}{x+k}\right)^{2} \\
& +\frac{a^{2}}{2}\left(\frac{-2 \beta^{2}(\alpha x+\gamma)}{(\alpha x+\beta y+\gamma)^{3}}\right)\left(-\frac{\alpha x+\gamma}{\alpha x+\beta y+\gamma}+\frac{y}{x+k}\right) \\
= & -\frac{2 a^{2} \alpha \beta(\alpha x+\gamma)}{(\alpha x+\beta y+\gamma)^{3}}+\frac{a^{2} \beta^{2}(\alpha x+\gamma)^{2}}{(\alpha x+\beta y+\gamma)^{4}}+\frac{a^{2}}{2(x+k)^{2}}+\frac{a^{2} \beta(\alpha x+\gamma)}{(\alpha x+\beta y+\gamma)^{2}(x+k)} \\
& -\frac{a^{2} \beta^{2} y(\alpha x+\gamma)}{2(\alpha x+\beta y+\gamma)^{3}(x+k)} .
\end{aligned}
$$


We have

$$
\begin{aligned}
\frac{\partial^{2} \phi}{\partial y^{2}} \leq & -\frac{2 a^{2} \alpha \beta(\alpha x+\gamma)}{(\alpha x+\beta y+\gamma)^{3}}-\frac{a^{2} \beta^{2} y(\alpha x+\gamma)}{2(\alpha x+\beta y+\gamma)^{3}(x+k)}+\frac{a^{2} \beta^{2}}{(\alpha x+\gamma)^{2}} \\
& +\frac{a^{2}}{2(x+k)^{2}}+\frac{a^{2} \beta}{(\alpha x+\gamma)(x+k)} \\
\leq & -\frac{a^{2} \beta(\alpha x+\gamma)}{2(\alpha x+\beta y+\gamma)^{3}(x+k)}(4 \alpha(x+k)+\beta y)+\frac{a^{2} \beta^{2}}{(\alpha x+\gamma)^{2}} \\
& +\frac{a^{2}}{2(x+k)^{2}}+\frac{a^{2} \beta}{(\alpha x+\gamma)(x+k)} .
\end{aligned}
$$

We note, using (5.1), that for $(x, y)$ in $A$

$$
\frac{1}{a^{2}} \frac{\partial^{2} \phi}{\partial y^{2}} \leq-\frac{2 k \beta^{2}}{\left(\alpha L_{1}+\gamma\right)^{3}(1+k)}+\frac{\beta^{2}}{\gamma^{2}}+\frac{1}{2 k^{2}}+\frac{\beta}{k \gamma} .
$$

If (5.3) holds, then

$$
\frac{\partial^{2} \phi}{\partial y^{2}} \leq 0
$$

Hence, $\partial \phi / \partial y$ is strictly decreasing in $\mathbb{R}_{+}$, with respect to $y$.

Now

$$
\begin{aligned}
\left.\frac{\partial \phi}{\partial y}\right|_{y=0} & =\frac{a^{2} \alpha}{\alpha x+\gamma}-\frac{a^{2}}{2}\left(\frac{\beta}{\alpha x+\gamma}+\frac{1}{x+k}\right) \\
& =\frac{a^{2}}{2(\alpha x+\gamma)(x+k)}((\alpha-\beta) x+k(2 \alpha-\beta)-\gamma) .
\end{aligned}
$$

In $A$, all solutions satisfy $0 \leq x \leq 1$, and from (5.1)

$$
(\alpha-\beta) x+k(2 \alpha-\beta)-\gamma \leq(\alpha-\beta)+k(2 \alpha-\beta)-\gamma,
$$

then, if (5.4) holds, $\left.(\partial \phi / \partial y)\right|_{y=0} \leq 0$ in $\mathbb{R}_{+}$. Hence, $\phi(x, y)$ is strictly decreasing in $\mathbb{R}_{+}$. This yields $\phi(x, y)<D(x, 0)$ for $(x, y) \in A$; that is, using (5.1), we get

$$
\begin{aligned}
\phi(x, y) & <-a\left(\alpha x^{*}+\beta y^{*}+\gamma\right)+\frac{a^{2}}{4} \\
& <-a\left(\beta\left(x^{*}+y^{*}\right)+\gamma-\frac{a}{4}\right) \\
& <-a\left(\beta L_{1}+\gamma-\frac{a}{4}\right) .
\end{aligned}
$$


Consequently, due to (5.5),

$$
\phi(x, y)<0, \quad \forall(x, y) \in A \text {. }
$$

It follows that if the hypotheses of Theorem 5.1 are satisfied, then $d V / d t<0$ along all trajectories in the first quadrant except $\left(x^{*}, y^{*}\right)$; so $P^{*}\left(x^{*}, y^{*}\right)$ is globally asymptotically stable.

\section{Conclusion}

The Beddington-DeAngelis functional response admits a range of dynamics which include the possibilities of extinction, persistence, and stable or unstable equilibria. The criteria for persistence are the same as for systems with a Holling-type 2 response.

The future research will complete the qualitative analysis by studying the limit cycles of the model. It will also contain the numerical simulations to justify the obtained results.

\section{References}

[1] Y. Takeuchi, Global Dynamical Properties of Lotka-Volterra Systems, World Scientific, Singapore, 1996.

[2] R. Arditi and L. R. Ginzburg, "Coupling in predator-prey dynamics: ratio-dependence," Journal of Theoretical Biology, vol. 139, pp. 311-326, 1989.

[3] S. Ruan and D. Xiao, "Global analysis in a predator-prey system with nonmonotonic functional response," SIAM Journal on Applied Mathematics, vol. 61, no. 4, pp. 1445-1472, 2001.

[4] D. Xiao and S. Ruan, "Global dynamics of a ratio-dependent predator-prey system," Journal of Mathematical Biology, vol. 43, no. 3, pp. 268-290, 2001.

[5] J. R. Beddington, "Mutual interference between parasites or predators and its effect on searching efficiency," Journal of Animal Ecology, vol. 44, pp. 331-340, 1975.

[6] R. S. Cantrell and C. Cosner, "On the dynamics of predator-prey models with the BeddingtonDeAngelis functional response," Journal of Mathematical Analysis and Applications, vol. 257, no. 1, pp. 206-222, 2001.

[7] D. L. DeAngelis, R. A. Goldstein, and R. V. OŃeil, “A model for trophic inter-action,” Ecology, vol. 56, pp. 881-892, 1975.

[8] J. K. Hale and P. Waltman, "Persistence in infinite-dimensional systems," SIAM Journal on Mathematical Analysis, vol. 20, no. 2, pp. 388-395, 1989.

[9] M. L. Rosenzweig, "Paradox of enrichment: destabilization of exploitation ecosystems in ecological time," Science, vol. 171, no. 3969, pp. 385-387, 1971.

[10] R. M. May, Stability and Complexity in Model Ecosystems, Princeton University Press, Princeton, NJ, USA, 1973.

[11] T. Royama, "A comparative study of models for predation and parasitism," Researches on Population Ecology, vol. 13, supplement 1, pp. 1-91, 1971.

[12] C. Cosner, D. L. Deangelis, J. S. Ault, and D. B. Olson, "Effects of spatial grouping on the functional response of predators," Theoretical Population Biology, vol. 56, no. 1, pp. 65-75, 1999.

[13] M. A. Aziz-Alaoui and M. D. Okiye, "Boundedness and global stability for a predator-prey model with modified leslie-gower and holling-type I schemes," Applied Mathematics Letters, vol. 16, no. 7, pp. 1069-1075, 2003.

[14] M. A. Aziz-Alaoui and M. Daher Okiye, "On the dynamics of a predator-prey model with the hollingtanner functional response," in Proceedings of the 5th European Conference on Mathematical and Theoretical Biology (ESMTB '02), pp. 1069-1075, 2002.

[15] A. F. Nindjin, M. A. Aziz-Alaoui, and M. Cadivel, "Analysis of a predator-prey model with modified leslie-gower and Holling-type II schemes with time delay," Nonlinear Analysis: Real World Applications, vol. 7, no. 5, pp. 1104-1118, 2006. 
[16] M. A. Aziz-Alaoui, "Study of a Leslie-Gower-type tritrophic population model," Chaos, Solitons and Fractals, vol. 14, no. 8, pp. 1275-1293, 2002.

[17] H. I. Freedman and S. G. Ruan, “Uniform persistence in functional-differential equations," Journal of Differential Equations, vol. 115, no. 1, pp. 173-192, 1995.

[18] H. I. Freedman, S. G. Ruan, and M. X. Tang, "Uniform persistence and flows near a closed positively invariant set," Journal of Dynamics and Differential Equations, vol. 6, no. 4, pp. 583-600, 1994.

[19] P. Y. H. Pang and M. Wang, "Strategy and stationary pattern in a three-species predator-prey model," Journal of Differential Equations, vol. 200, no. 2, pp. 245-273, 2004. 


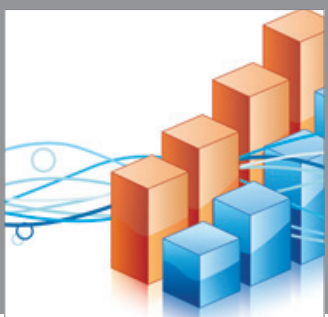

Advances in

Operations Research

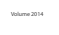

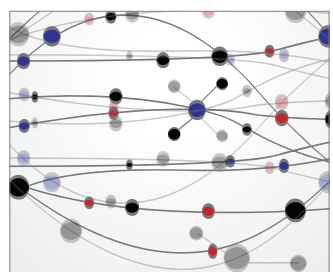

\section{The Scientific} World Journal
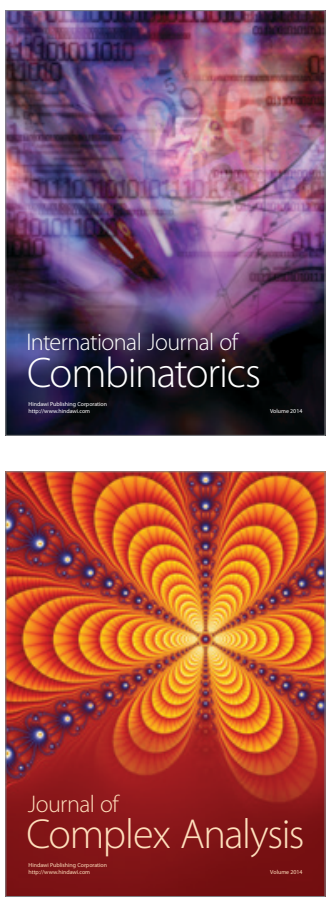

International Journal of

Mathematics and

Mathematical

Sciences
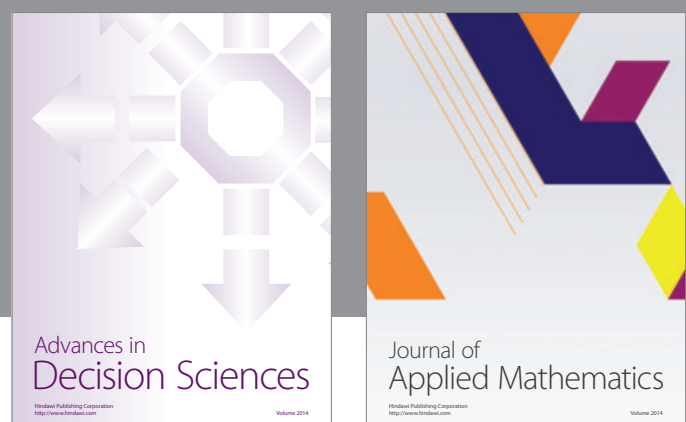

Journal of

Applied Mathematics
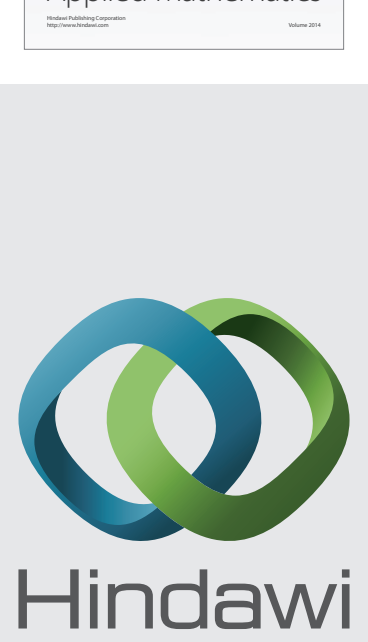

Submit your manuscripts at http://www.hindawi.com
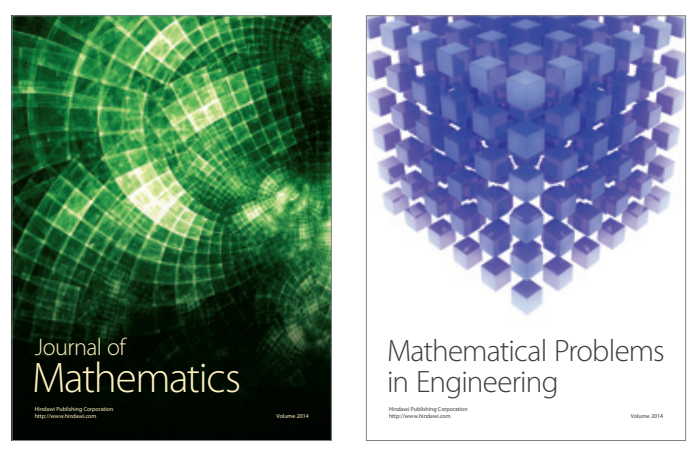

Mathematical Problems in Engineering
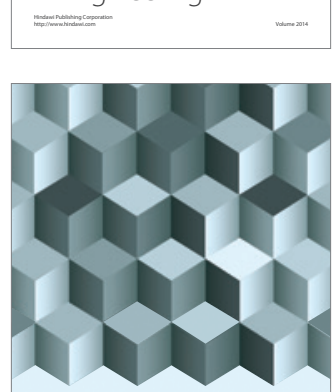

Journal of

Function Spaces
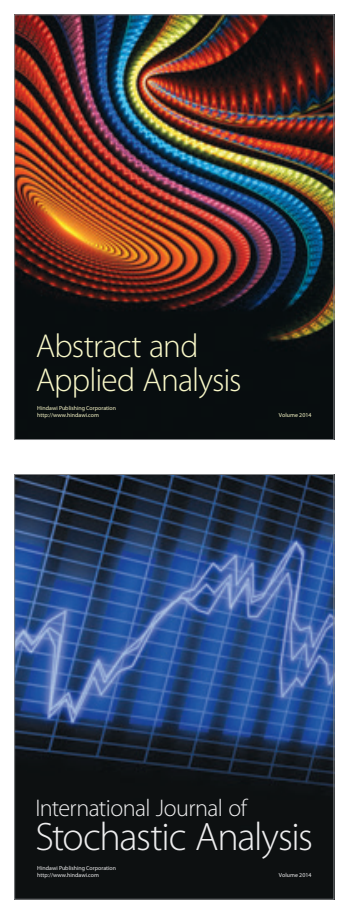

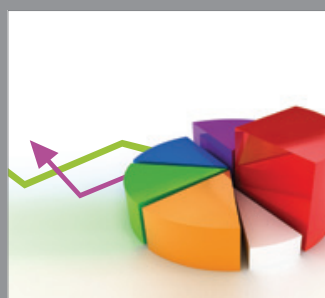

ournal of

Probability and Statistics

Promensencen
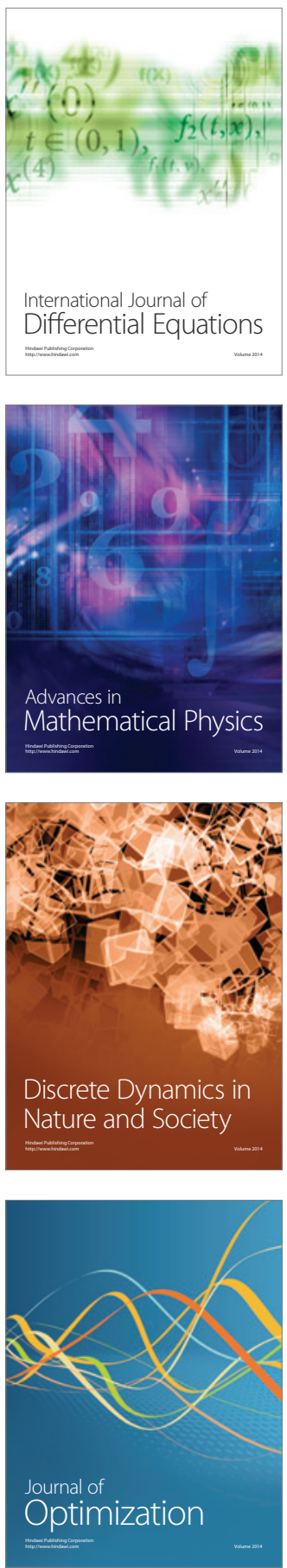